\title{
Influence of Self-efficacy Improvement on Online Learning Participation
}

\author{
https://doi.org/10.3991/ijet.v17i01.28719 \\ Li Geng \\ Academy of Sport Education, Xi'an Physical Education University, Xi'an, China \\ 102015@tea.xaipe.edu.cn
}

\begin{abstract}
More and more online learning apps are emerging, thanks to the development of Internet plus education and online learning platforms. Learning efficacy is the leading impactor of online learning participation. To avoid inefficiency and poor effect of online learning, it is necessary to explore the theory on the relationship between self-efficacy improvement and online learning participation. This paper examines the influence of self-efficacy improvement on online learning participation. Firstly, a general normal distribution map was drawn for self-efficacy. Then, a prediction model was established for participation based on the series of online learning behaviors. In addition, the k-means clustering (KMC) algorithm was optimized by information entropy, and the flow of the improved KMC was explained. The proposed model was proved valid through experiments.
\end{abstract}

Keywords_-self-efficacy, online learning behaviors, learning participation, cluster analysis

\section{Introduction}

With the development of Internet plus education and online learning platforms, the dominance of classroom learning is being replaced with the combination between classroom learning and online learning [1-6]. Learning refers to the process that learners actively construct knowledge and experience [7-11]. To avoid inefficiency and poor effect of online learning, and to fully motivate the initiative of students, it is necessary to effectively improve their online learning participation [12-15]. Many factors could affect online learning participation. One of these factors is learning efficacy [16-20]. The theoretical research on the relationship between self-efficacy improvement and student online learning participation helps teachers to understand the status quo of teaching, and enhance the teaching effect.

Massive open online courses (MOOCs) have created a highly individualized and dynamic learning environment for higher education. However, the development of MOOCs is hindered by low completion rate. Susanti et al. [21] surveyed mediating role of online academic tenacity between online learning and self-efficacy, and discovered the significant mediating effect of commitment on the relationship between the behaviors, emotions, and cognitive participation of online learning. The outbreak 
of coronavirus COVID-19 brings new challenges to education, and presents a revolutionary opportunity for integrating the construction of information society. The resulting new learning model, i.e., family-based online learning, raises new requirements on college students. From the perspective of students' self-efficacy, Liu et al. [22] extended online learning to four dimensions, namely, sense of effort, sense of control, sense of participation, and sense of environment, and analyzed such five factors as learning attitude, learning strategy, learning interaction, learning evaluation, and learning environment, and put forward four strategies for improving learning effect.

Through literature review, surveys, and quasi-experiments, Krouska et al. [23] conducted theoretical analysis, scale preparation, experimental intervention, and effect testing to clarify the definition and structure of college students' self-efficacy of online learning, and explored some practical improvement strategies. Shi et al. [24] discussed how intelligent classroom teaching affects learning input, and online selfefficacy, and demonstrated that the students receiving intelligent classroom teaching have higher learning participation and online self-efficacy than those receiving traditional teaching. Peechapol et al. [25] reviewed the research in the past 12 years, which tackle the factors affecting self-efficacy in online learning environment, and the sources of self-efficacy in that environment. They further designed an online learning environment that guides learners to improve their self-efficacy.

Overall, the existing studies rarely talk about the correlations between self-efficacy and online learning participation. Only a few scholars have theoretically discussed the two factors independently. The few studies on their correlations are too general, without any detailed deliberation. Therefore, this paper examines the influence of selfefficacy improvement on online learning participation. The contents mainly evolve around two themes: (1) plotting a normal distribution map of self-efficacy in online learning, and building a prediction model for participation based on the series of online learning behaviors; (2) optimizing the k-means clustering (KMC) algorithm based on information entropy, and clarifying the flow of the improved algorithm. Experimental results demonstrate the effectiveness of our model.

\section{Feature extraction}

Self-efficacy, the sense of learning effectiveness, refers to students' cognition and belief of the degree of completion for learning goals. The self-efficacy of online learning can be understood as the manifestation of self-efficacy through the online learning process, that is, the students' belief of improving learning behavior control and learning quality during online learning. The online learning self-efficacy is closely related to most learning activities. The level of online learning self-efficacy directly affects the students' confidence in participating in online learning activities, and their degree of completion for learning tasks. Figure 1 shows the normal distribution map of online learning self-efficacy. It can be observed that the development level of online learning self-efficacy obeys normal distribution.

Based on the series of online learning behaviors, this paper predicts online learning participation by classifying the recent online learning behaviors, such as login, topic 
Paper-Influence of Self-efficacy Improvement on Online Learning Participation

discussion, online test, and MOOC watching. Figure 2 shows the structure of the participation prediction model.

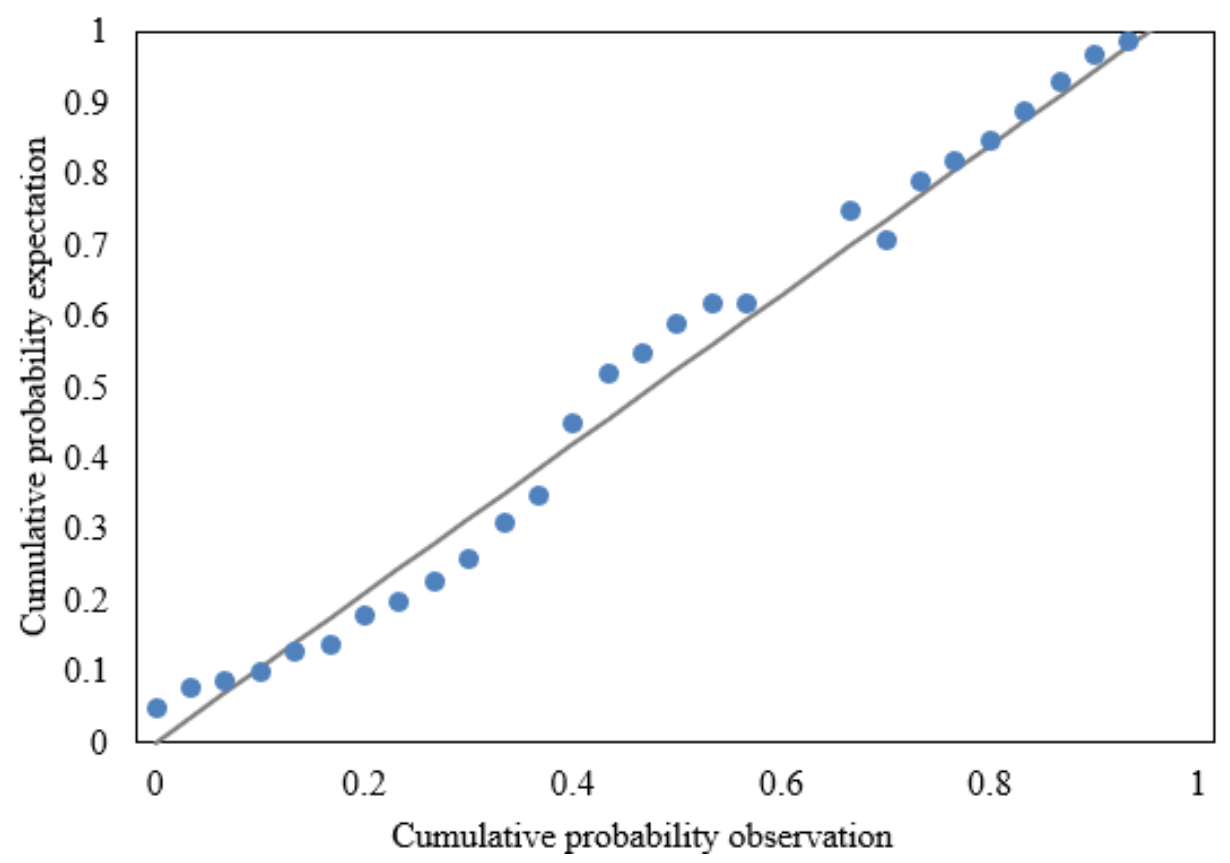

Fig. 1. Normal distribution map of online learning self-efficacy 


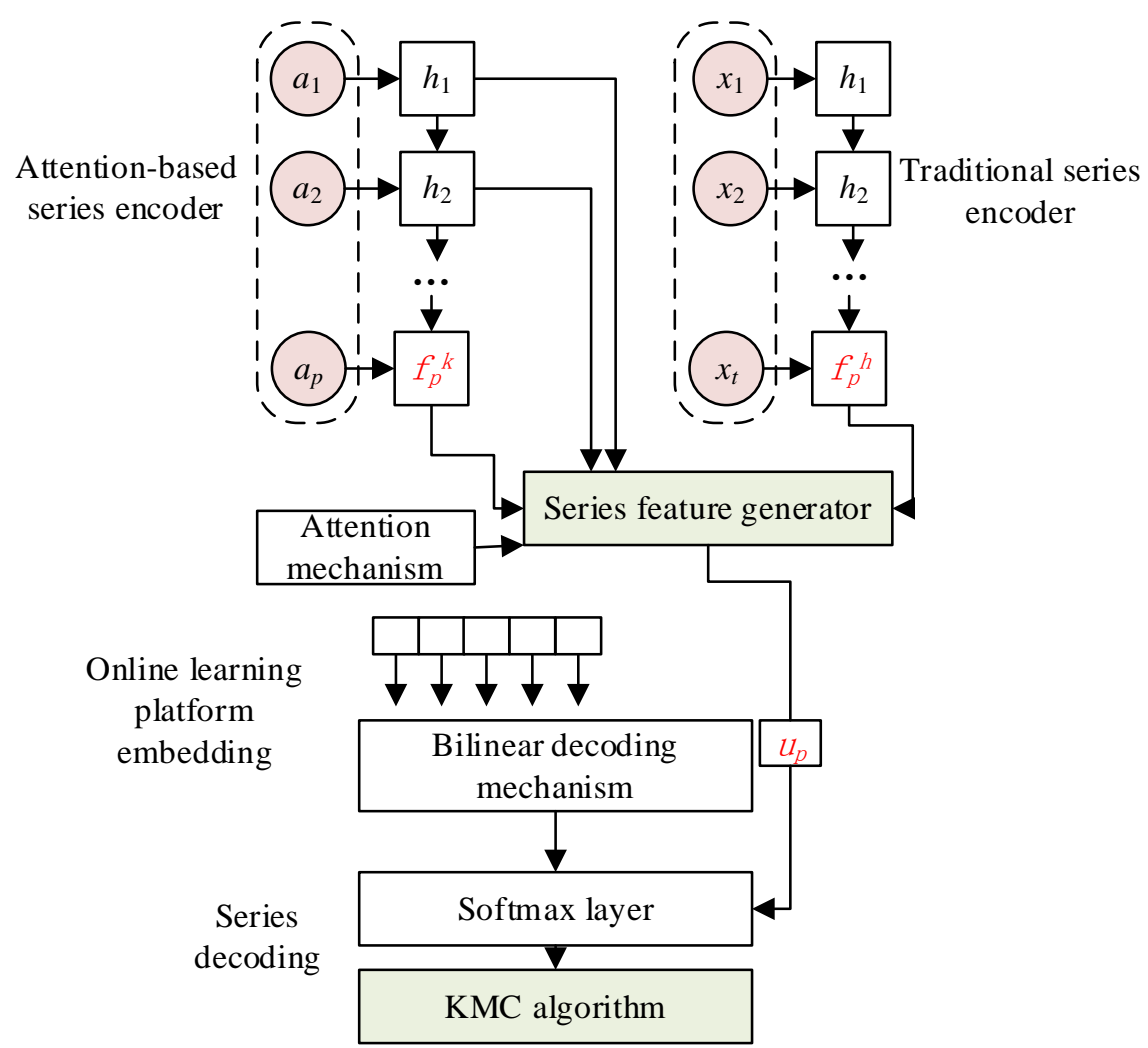

Fig. 2. Structure of participation prediction model

In the first phase of series-based prediction of learning participation, this paper constructs an attention-based hybrid encoder-decoder model, and uses the model to extract the features of the online learning behavior series under self-efficacy improvement. It is assumed that the upper and lower encoders receive the historical data of online learning behaviors $a=\left[a_{1}, a_{2}, \ldots, a_{p-1}, a_{p}\right]$, and output the hidden state $f_{i}$ of the online learning behavior series.

The specific steps of the proposed algorithm are as follows: For each behavior series $a_{i}$, the gated unit of the model receives the historical online learning behavior series, and outputs the linear transform between the hidden state $f_{p}{ }_{p}$ of the current online learning behavior series, and that $f_{p-1}$ of the previous online learning behavior series. The update gate $c_{p}$ controls how much information in $f_{p-1}$ should be forgotten and how much information in $f^{\prime}$, should be memorized:

$$
c_{p}=\tau\left(Q^{(c)} a_{p}+V^{(c)} f_{p-1}\right)
$$

The reset gate $s_{p}$ determines that the memory of the previous moment should be preserved: 


$$
s_{p}=\tau\left(Q^{(s)} a_{p}+V^{(s)} f_{p-1}\right)
$$

The new network unit can be expressed as:

$$
f_{p}^{\prime}=\tan \left(Q a_{p}+s_{p} \oplus f_{p-1}\right)
$$

The hidden state can be expressed as:

$$
f_{p}=c_{p} \oplus f_{p-1}+\left(1-c_{p}\right) \oplus f_{p}^{\prime}
$$

The above analysis shows that the linear transform between $f_{p}^{\prime}$ and $f_{p-1}$ is a linear interpolation between the hidden states of the current and previous online learning behavior series. The hidden state $f_{p}$ finally outputted by the encoders carries most of the information in the initial online learning behavior series.

The features of online learning behavior series can be characterized by $f_{p}$, i.e., $f_{p}^{h}$ as:

$$
u_{p}^{h}=f_{p}=f_{p}^{h}
$$

Not every recent online learning behavior is associated with the final learning effect. During participation prediction, the proposed model is expected to interact more with the behaviors related to learning quality and learning effect. Therefore, this paper extracts the above assumption based on the attention mechanism, and constructs an attention-based series encoder:

$$
u_{p}^{k}=\sum^{p} \gamma_{p i} f_{i}
$$

Where, $u_{p}^{k}$ is the context vector; $\gamma_{p i}$ is the weighting factor. The context factor can be calculated from $\gamma_{p i}$ and hidden states $f_{1}-f_{p}(1 \leq i \leq p)$. Let $\varphi$ be the sigmoid function that transforms $f_{p}$ and $f_{i}$ to a latent space. Then, the attention-based mechanism can be described as:

$$
\gamma_{p i}=\phi\left(Q_{\gamma}\left[f_{p} ; f_{i}\right]\right)
$$

Then, all hidden states are weighed and summarized. The sum is adopted to characterize the features of learning behavior series. To better understand $u_{p}^{k}$, this paper describes $f_{i}$ as the final hidden state $f_{p}$ at moment $p$, i.e., $f_{p}^{k}$. Hence, $u_{p}^{k}$ can be optimized as:

$$
u_{p}^{k}=\sum^{p} \gamma_{p i} f_{i}=\sum^{p} \gamma_{p i} f_{p}^{k}
$$

It can be seen that $f_{p}^{h}$ is incorporated into $u_{p}^{h}$, while $f_{p}^{k}$ and $\gamma_{p i}$ are incorporated into $u_{p}^{k}$. Together, $u_{p}^{h}$ and $u_{p}^{k}$ represent the online learning behavior series of the proposed 
model. The difference between the series-based encoder $f_{p}^{h}$ and the attention-based encoder $f_{p}^{k}$ lies in that: the final hidden state of the former is responsible for encoding the entire online learning behavior series, while the latter is responsible for computing the attention weight of the previous hidden state. In the hybrid scheme, the two encoders can be expressed as $u_{p}$, i.e., a series generator pieced up from $u_{p}^{h}$ and $u_{p}^{k}$ :

$$
u_{p}=\left[u_{p}^{h} ; u_{p}^{k}\right]=\left[f_{p}^{h} ; \sum^{p} \gamma_{p i} f_{p}^{k}\right]
$$

Let $|G|$ be the embedding dimension of online learning platform, which maps each behavior vector to the low-dimensional space; $|F|$ be the dimension of series state; $\psi$ be a $|G|^{*}|F|$ matrix. To better predict online learning participation, this paper calculates the similarity score $X Z_{i}$ by applying the selective bilinear decoding mechanism between the hidden state of the current online learning behavior series and the online learning platform:

$$
X Z_{i}=e m b_{i}^{n} \psi u_{p}
$$

The softmax layer receives the similarity score of online learning platform, and decodes the proposed model with the probability of acquiring the deep behavior features of online learning.

\section{Learning participation classification}

For participation prediction, an important step is to establish the evaluation indices for behavior features by analyzing the online learning behavior series. The evaluation criteria, such as positivity, indifference, inactivity, and nonparticipation, can be determined based on the prediction goals. Based on the deep behavior features obtained in the preceding section, this section attempts to classify the students' learning participation with the improved KMC algorithm.

The $m$ behavior features of sample set $R$ of online learning behavior features, and $K$ classes are imported to the algorithm. The sample set $R$ can be expressed as:

$$
R=\left\{a_{1}, a_{2}, \ldots a_{m}\right\}, K=\left\{u_{1}, u_{2}, \ldots, u_{k}\right\}
$$

The algorithm outputs $K$ classes that satisfy the demand. The algorithm is realized in the following steps:

Step 1 . Randomly choose $K$ behavior features from $R$, and treat them as the initial cluster heads.

Step 2. Based on the mean of each behavior feature, compute the Euclidean distance $\varepsilon$ from each sample to each initial cluster head, and re-classify the samples based on the minimum $\varepsilon$. The Euclidean distance $\varepsilon\left(a_{i}, a_{j}\right)$ between two $T$-dimensional behavior features $a_{i}=\left(a_{i 1}, a_{i 2}, \ldots, a_{i t}\right)$ and $a_{j}=\left(a_{j 1}, a_{j 2}, \ldots, a_{j t}\right)$ can be defined as: 


$$
\varepsilon\left(a_{i}, a_{j}\right)=\sqrt{\left(a_{i 1}-a_{j 1}\right)^{2}+\left(a_{i 2}-a_{j 2}\right)^{2}+\ldots\left(a_{i t}-a_{j t}\right)^{2}}
$$

The mean distance between all samples can be calculated by:

$$
\operatorname{MEA}(R)=\frac{2}{m(m-1)} \times \sum_{i \neq j, j, j=1}^{m} \varepsilon\left(a_{i}, a_{j}\right)
$$

Step 3. Re-calculate the mean of each behavior feature.

Step 4. Repeat Steps 2 and 3 until the objective function value tends to be stable or falls below the preset threshold. Let $u_{i}$ be the centroid of behavior features in the same class. Then, the objective function, i.e., the squared error criterion, can be given by:

$$
\delta_{i}=\sqrt{\frac{\sum_{i=1}^{m_{i}}\left(a_{i}-u_{i}\right)^{2}}{\left|U_{i}\right|-1}}
$$

The centroid $u_{i}$, i.e., the head of cluster $i$, can be calculated by:

$$
u_{i}=\frac{1}{\left|U_{i}\right|} \sum_{a_{j} \in \Psi_{i}} a_{j}
$$

Where, $c_{i} u_{i}$ is the center of cluster $i$; $\left|U_{i}\right|$ be the number of features in cluster $U_{i}$.

Step 5. End the algorithm and obtain $K$ clusters.

Figure 3 shows the main steps of the KMC algorithm.

The traditional KMC algorithm has a large stochasticity and a high computing overhead. To solve these defects, this paper optimizes the KMC algorithm based on information entropy. Firstly, the information entropy was calculated for the behavior feature samples. Then, a weight was assigned to the Euclidean distance from each sample to each initial cluster head. After that, the criterion function value was computed, and the initial cluster heads were determined. On this basis, the behavior feature sample set was fully clustered. 


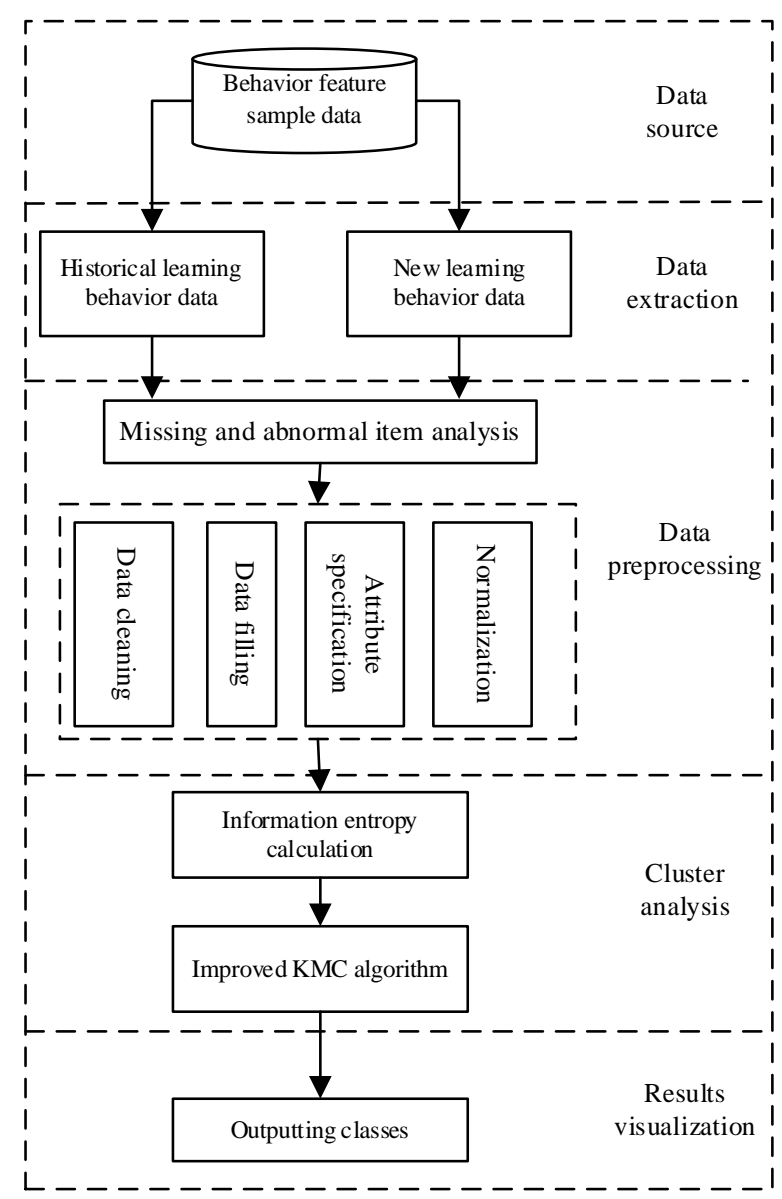

Fig. 3. Main steps of original KMC algorithm

The information entropy is calculated as follows: Let $r=\left\{a_{1}, a_{2}, \ldots, a_{m}\right\}$ be the behavior feature sample set; $Q G_{i}=Q G\left[A=a_{i}\right]$ be the probability density. Then, the selfinformation volume of a behavior can be expressed as:

$$
S F\left(a_{i}\right)=\log \frac{1}{Q G_{i}}
$$

The information entropy of the behavior feature sample set can be expressed as:

$$
S D(A)=\sum_{i} Q G_{i} \log \frac{1}{Q G_{i}}(i=1,2, \ldots, m)
$$

The information entropy, as a measure of information volume, is positively correlated with uncertainty. 
After analyzing the contribution of each behavior feature to the clustering of behavior feature samples, a weight is calculated for each behavior feature, and the Euclidean distance between samples is calculated again to complete clustering. The entropy-based feature weighting is implemented in the following steps:

Step 1. Let $n$ be the dimensionality of behavior feature samples, and $m$ be the number of samples. Then, the behavior feature matrix can be established as:

$$
X=\left[\begin{array}{cccc}
x_{11} & x_{12} & \cdots & x_{1 m} \\
x_{21} & x_{22} & \cdots & x_{2 m} \\
\vdots & \vdots & & \vdots \\
x_{n 1} & x_{n 2} & \cdots & x_{n m}
\end{array}\right]
$$

Step 2. Compute the weight of each behavior feature, i.e., the ratio of the behavior feature in dimension $j$ to the behavior feature of sample $i$. firstly, normalize sample data to the interval $[0,1]$. Let $x_{i j}$ be the value of behavior feature, with $i=1,2, \ldots, n$, and $j=1,2, \ldots, m$. Then, the behavior feature ratio $\Phi_{i j}$ can be calculated by:

$$
\Phi_{i j}=x_{i j} / \sum_{i=1}^{m} x_{i j}
$$

Step 3. The entropy of the behavior feature in dimension $j$ can be calculated by:

$$
S D_{j}=-\frac{1}{\ln m} \sum_{i=1}^{m} \Phi_{i j} \ln \Phi_{i j}
$$

If $\Phi_{i j}=0$, then $\Phi_{i j} 1 n \Phi_{i j}=0$. If all behavior features are equal, for a given $j$. Then, we have:

$$
\Phi_{i j}=x_{i j} / \sum_{i=1}^{\Phi} x_{i j}=1 / m
$$

Step 4. The diversity factor for the behavior feature in dimension $j$ can be calculated by:

$$
\mu_{j}=1-S D_{j}
$$

For a given $j$, the smaller the entropy $S D_{j}$ of behavior feature, the greater the $\mu_{j}$, and the more important the corresponding behavior feature. The inverse is also true. If $S D_{j}=1$, then $\mu_{j}=0$, and the behavior feature has a negligeable contribution to clustering.

Step 5 . The behavior feature in dimension $j$ can be calculated by:

$$
\alpha_{j}=\mu_{j} / \sum_{j=1}^{n} \mu_{j}
$$

Step 6. Let $\alpha_{t}$ the weight of the behavior feature in dimension $t$. After the weighting, the Euclidean distance can be calculated by: 


$$
\varepsilon_{\alpha}=\left(a_{i}, a_{j}\right)=\sqrt{\sum_{t=1}^{n} \alpha_{t}\left(a_{i t}-a_{j t}\right)^{2}}
$$

The above formula essentially scales up or down behavior features properly according to their weights, such that the behavior features with a large weight contribute more to clustering, and those with a small weight contribute less to clustering.

Step 7. Let $\delta_{i}$ be the standard error of the weights assigned to class $i$; $\left|U_{j}\right|$ be the number of behavior features in $U_{j}$. Taking $\delta_{i}$ as the standard measuring function, the target value of each weighted class can be expressed as:

$$
\delta_{i}=\sqrt{\frac{\sum_{x_{i} \in \psi_{j}} \varepsilon_{\alpha}\left(x_{i}, u\left(U_{j}\right)\right)}{\left|U_{j}\right|-1}}
$$

The greater the $\delta_{i}$, the smaller the similarity between behavior features in the same class, and the more dispersed the behavior features. The smaller the $\delta_{i}$, the more concentrated the samples. In the latter case, the centroid of the class of a sample can better reflect the classification plane for behavior features. Figure 4 shows the steps of information entropy-based KMC algorithm.

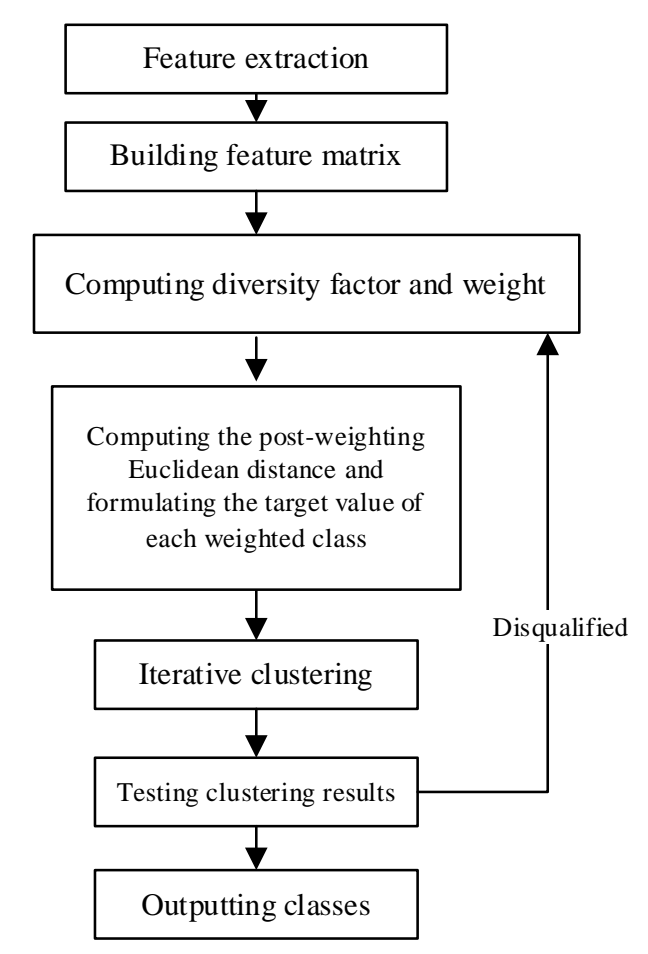

Fig. 4. Steps of information entropy-based KMC algorithm 


\section{$4 \quad$ Experiments and results analysis}

Table 1 lists the online learning self-efficacy of students in different age groups. In the age group of $16-18$, more than $91 \%$ have a relatively high self-efficacy, including $68 \%$ on the medium level, and $23 \%$ on the high level. In the age group of $19-21$, more than $92 \%$ have a relatively high self-efficacy, including $56 \%$ on the medium level, and $36 \%$ on the high level. In the age group of $22-24$, more than $93 \%$ have a relatively high self-efficacy, including $74 \%$ on the medium level, and $16 \%$ on the high level. In the age group of 25 and above, all students have a relatively high self-efficacy, including $41 \%$ on the medium level, and $56 \%$ on the high level. Therefore, older students are more capable of completing various learning tasks during online learning, and more confident in learning.

Table 2 shows the results of correlation analysis on online learning self-efficacy and classroom learning self-efficacy. The results show that the p-value of the correlations between the two self-efficacies was 0.001 , smaller than 0.1 . Hence, there is a significant correlation between online learning self-efficacy and classroom learning self-efficacy.

Table 1. Online learning self-efficacy of students in different age groups

\begin{tabular}{|l|c|c|c|c|c|c|c|c|}
\hline \multirow{2}{*}{$\begin{array}{c}\text { Age } \\
\text { group }\end{array}$} & \multicolumn{2}{|c|}{ 16-18 } & \multicolumn{2}{c|}{ 19-21 } & \multicolumn{2}{c|}{ 22-24 } & \multicolumn{2}{c|}{ 25 and above } \\
\cline { 2 - 9 } & Number & Proportion & Number & Proportion & Number & Proportion & Number & Proportion \\
\hline $\begin{array}{l}0-20 \\
\text { Low }\end{array}$ & 5 & $6 \%$ & 4 & $8 \%$ & 2 & 1 & $/$ & $/$ \\
\hline $\begin{array}{l}20-40 \\
\text { Medium }\end{array}$ & 32 & $68 \%$ & 24 & $56 \%$ & 8 & $74 \%$ & 6 & $41 \%$ \\
\hline $\begin{array}{l}40-60 \\
\text { High }\end{array}$ & 12 & $23 \%$ & 14 & $36 \%$ & 5 & $16 \%$ & 8 & $56 \%$ \\
\hline
\end{tabular}

Table 2. Correlation between online learning self-efficacy and classroom learning self-efficacy

\begin{tabular}{|l|c|c|c|c|}
\hline \multirow{2}{*}{} & \multicolumn{2}{|c|}{ Online learning self-efficacy } & \multicolumn{2}{c|}{ Classroom learning self-efficacy } \\
\cline { 2 - 5 } & Correlation & Significance & Correlation & Significance \\
\hline Online learning self-efficacy & 1 & & $0.618^{* *}$ & 0.001 \\
\hline Classroom learning self-efficacy & $0.618^{* *}$ & 0.001 & 1 & \\
\hline
\end{tabular}

Table 3 presents the descriptive statistics of different types of online learning participation. Five types of online learning participation were surveyed, including conventional participation, propelled participation, spontaneous participation, extended participation, and knowledge-centered participation. The statistics in Table 3 shows that the min, max, mean and SD of conventional participation were $5,15,35.25$, and 2.582 , respectively; the min, max, mean and SD of propelled participation were 2,18 , 26.35, and 6.258, respectively; the min, max, mean and SD of spontaneous participation were 4, 17, 21.38, and 4.296, respectively; the min, max, mean and SD of extended participation were $1,12,19.28$, and 4.287, respectively; the min, max, mean 
and SD of knowledge-centered participation were 7, 16, 10.19, and 2.364, respectively.

Table 3. Descriptive statistics of different types of online learning participation

\begin{tabular}{|l|c|c|c|c|c|}
\hline & $\begin{array}{c}\text { Conventional } \\
\text { participation }\end{array}$ & Propelled participation & $\begin{array}{c}\text { Spontaneous } \\
\text { participation }\end{array}$ & Extended participation & $\begin{array}{c}\text { Knowledge- } \\
\text { centered } \\
\text { participation }\end{array}$ \\
\hline Min & 5 & 2 & 4 & 1 & 7 \\
\hline Max & 15 & 18 & 17 & 12 & 16 \\
\hline Mean & 35.25 & 26.35 & 21.38 & 19.28 & 10.19 \\
\hline SD & 2.582 & 6.258 & 4.296 & 4.287 & 2.364 \\
\hline
\end{tabular}

Note: Min, max, mean, and SD are short for minimum, maximum, mean value, and standard deviation, respectively.

By the mean values, conventional participation covers the greatest proportion of online learning students, followed in turn by propelled participation, spontaneous participation, extended participation, and knowledge-centered participation. Knowledge-centered participation involves the fewest number of online learning students. Overall, the five types of participation differ very slightly in mean value. According to the min values of different types of participation, some students very rarely engage in extended and propelled participation. Judging by the SDs, the students differ insignificantly between spontaneous participation and extended participation, but differ greatly between conventional participation and propelled participation.

The conventional and our improved KMCs were separated applied to cluster the sample set of online learning behavior features and the sample set of classroom learning behavior features. The clustering results are recorded in Table 4. The clustering effect was measured by the classification accuracy of participation, i.e., the number of correctly clustered instances as a percentage of the total number of instances. The classification accuracy of the sample set of classroom learning behavior features increased from $82.14 \%$ of the conventional KMC to $89.32 \%$ of the improved KMC; the classification accuracy of the sample set of online learning behavior features increased from $90.21 \%$ of the conventional KMC to $96.47 \%$ of the improved KMC. The comparison demonstrates the effectiveness of our improved algorithm.

Table 4. Results of different clustering algorithms on different sample sets

\begin{tabular}{|l|c|c|c|c|c|c|c|c|}
\hline $\begin{array}{c}\text { Sample set } \\
\text { number }\end{array}$ & \multicolumn{2}{|c|}{ Classes } & $\mathbf{1}$ & $\mathbf{2}$ & $\mathbf{3}$ & $\mathbf{4}$ & $\mathbf{5}$ & Accuracy \\
\hline \multirow{2}{*}{1} & Conventional KMC & Number of instances & 111 & 113 & 114 & 111 & 112 & $82.14 \%$ \\
\cline { 2 - 8 } & Improved KMC & $\begin{array}{c}\text { Number of correctly clustered } \\
\text { instances }\end{array}$ & 95 & 94 & 97 & 96 & 102 & $89.32 \%$ \\
\hline \multirow{2}{*}{2} & Conventional KMC & Number of instances & 104 & 102 & 103 & 105 & 101 & $90.21 \%$ \\
\cline { 2 - 8 } & Improved KMC & $\begin{array}{c}\text { Number of correctly clustered } \\
\text { instances }\end{array}$ & 101 & 99 & 100 & 100 & 98 & $96.47 \%$ \\
\hline
\end{tabular}




\section{Conclusions}

To disclose the influence of self-efficacy improvement on online learning participation, this paper presents a general normal distribution map for self-efficacy, establishes a prediction model based on the series of online learning behaviors, optimizes the KMC algorithm, and specifies the flow of the improved KMC. Through experiments, the authors summarized the online learning self-efficacy of students in different age groups, analyzed the correlations between the online learning self-efficacy and classroom learning self-efficacy, collected the descriptive statistics on different types of participation, and compared the clustering results of different algorithms on different sample sets. The experimental results demonstrate the effectiveness of our improved algorithm.

\section{$6 \quad$ References}

[1] van Erven, T., Koolen, W.M., van der Hoeven, D. (2021). MetaGrad: Adaptation using multiple learning rates in online learning. arXiv preprint arXiv:2102.06622.

[2] Jin, D.M., Li, Y.P. (2020). A Teaching Model for College Learners of Japanese Based on Online Learning, International Journal of Emerging Technologies in Learning, 15(15): 162-175. https://doi.org/10.3991/ijet.v15i15.15929

[3] Lin, J., Zhou, D.X. (2017). Online learning algorithms can converge comparably fast as batch learning. IEEE transactions on neural networks and learning systems, 29(6): 23672378. https://doi.org/10.1109/TNNLS.2017.2677970

[4] Dixit, A., Shanthamallu, U.S., Spanias, A., Berisha, V., Banavar, M. (2018). Online machine learning experiments in HTML5. In 2018 IEEE Frontiers in Education Conference (FIE), 1-5. https://doi.org/10.1109/FIE.2018.8659113

[5] Weed, J., Perchet, V., Rigollet, P. (2016). Online learning in repeated auctions. In Conference on Learning Theory, 1562-1583.

[6] Chen, F.S., Ke, H.S., Chen, Y.C. (2020). Online Learning as a Panacea? An Empirical Study to Discuss Problem-Based Cooperative Learning in Taiwan, International Journal of Emerging Technologies in Learning, 15(18): 251-259. https://doi.org/10.3991/ijet.v15i $\underline{18.15079}$

[7] Kent, P., Mester, L., Steudle, K., Thayer, S., Van Port Fleet, M., Van Portfliet, R. (2016). Developing a knowledge management practice: One state's experience. TR News, 305: 3235. http://worldcat.org/issn/07386826

[8] Szczerbicki, E. (2016). Guest editorial: smart experience and knowledge engineering for optimization, learning, and classification/recommendation problems. Cybernetics and Systems, 47(1-2): 1-3. https://doi.org/10.1080/01969722. 2016.1128777

[9] Leake, D., Maguitman, A., Reichherzer, T. (2014). Experience-based support for humancentered knowledge modeling. Knowledge-based systems, 68: 77-87. https://doi.org/10. $\underline{1016 / j . k n o s y s .2014 .01 .013}$

[10] Fink, A., Vogt, S. (2014). Managing knowledge-intensive business processes by harnessing collective practical experience without codification. In International Conference on Subject-Oriented Business Process Management, 107-121. https://doi.org/10.1007/978-3319-06191-7 7 
[11] Ruiz, P.P., Foguem, B.K., Grabot, B. (2014). Generating knowledge in maintenance from Experience Feedback. Knowledge-Based Systems, 68: 4-20. https://doi.org/10.1016/j. knosys.2014.02.002

[12] Coll, C., Rochera, M.J., De Gispert, I. (2014). Supporting online collaborative learning in small groups: Teacher feedback on learning content, academic task and social participation. Computers \& Education, 75: 53-64. https://doi.org/10. 1016/j.compedu.2014.01.015

[13] Shaw, R.S. (2013). The relationships among group size, participation, and performance of programming language learning supported with online forums. Computers \& Education, 62: 196-207. https://doi.org/10.1016/j.compedu. 2012.11.001

[14] Cheng, G., Chau, J. (2016). Exploring the relationships between learning styles, online participation, learning achievement and course satisfaction: An empirical study of a blended learning course. British Journal of Educational Technology, 47(2): 257-278. https://doi. org/10.1111/bjet.12243

[15] Cheng, G., Chui, H.L. (2015). The greater the online participation, the better the learning achievement? A study of integrating Moodle into learning. In Technology in Education. Transforming Educational Practices with Technology (pp. 136-144). Springer, Berlin, Heidelberg. https://doi.org/10.1007/978-3-662-46158-7_14

[16] Yan, X., Hu, Z. (2016). Relationship between the on-line learning environment and students' learning self-efficacy. World Trans. on Engng. and Technol. Educ, 14(1): 31-38.

[17] Hong, J.C., Hwang, M.Y., Szeto, E., Tsai, C.R., Kuo, Y.C., Hsu, W.Y. (2016). Internet cognitive failure relevant to self-efficacy, learning interest, and satisfaction with social media learning. Computers in Human Behavior, 55: 214-222. https://doi.org/10.1016/ j.chb.2015.09.010

[18] Novinta, A.Y., Partana, C.F. (2021). Senior high school students' self-efficacy on learning acid-base. In Journal of Physics: Conference Series, 1806(1): 012205. https://doi.org/ $10.1088 / 1742-6596 / 1806 / 1 / 012205$

[19] Zhu, J., Wang, J., Wang, X., Gao, M., Guo, B., Gao, M., Xie, Z. (2021). Prediction of drug efficacy from transcriptional profiles with deep learning. Nature Biotechnology, 1-9. https://doi.org/10.1038/s41587-021-00946-z

[20] Kosycheva, M., Victorovna Tikhonova, E. (2021). Students' self-efficacy and motivation in emergency remote learning. In 2021 12th International Conference on E-Education, EBusiness, E-Management, and E-Learning, 157-162. https://doi.org/10.1145/3450148. $\underline{3450207}$

[21] Susanti, A., Diani, R., Satiarti, R.B., Munawaroh, R., Fujiani, D. (2021). Blended learning model: The effect on physics problem-solving skills viewed from self-efficacy. In Journal of Physics: Conference Series, 1796(1): 012014. https://doi.org/10.1088/1742-6596/1796/ $1 / 012014$

[22] Liu, H.H., Ye, Y.C., Jiang, H.L. (2021). Self-efficacy in home-based online learning environments. Journal of Internet Technology, 22(3): 557-567.

[23] Krouska, A., Troussas, C., Virvou, M. (2019). Using learning analytics to improve the efficacy of mobile authoring tools. In 2019 10th International Conference on Information, Intelligence, Systems and Applications (IISA), 1-5. https://doi.org/10.1109/IISA.2019. $\underline{8900726}$

[24] Shi, Y., Peng, C., Wang, S., Yang, H.H. (2018). The effects of smart classroom-based instruction on college students' learning engagement and internet self-efficacy. In International Conference on Blended Learning, 263-274. https://doi.org/10.1007/978-3-319$\underline{94505-7 \_21}$

[25] Peechapol, C., Na-Songkhla, J., Sujiva, S., Luangsodsai, A. (2018). An exploration of factors influencing self-efficacy in online learning: a systematic review. International Journal 
Paper-Influence of Self-efficacy Improvement on Online Learning Participation

of Emerging Technologies in Learning, 13(9): 64-86. https://doi.org/10.3991/ijet.v13i09. $\underline{8351}$

\section{Author}

Li Geng, Graduated from Northwest University of Politics and Law in 2006. Work at Xi'an Physical Education University. Engaged in the research of Ideological and Political Education, Student management.

Article submitted 2021-10-08. Resubmitted 2021-12-15. Final acceptance 2021-12-16. Final version published as submitted by the author. 